\title{
Transvaginal lymphatic embolization of the fistula between a pelvic lymphocele and the vaginal stump following radical hysterectomy and sentinel pelvic lymph node biopsy in a patient with cervical cancer: A case report
}

\author{
YEON JEE LEE ${ }^{1}$, IN JOON LEE ${ }^{2}$, SOHYUN PARK ${ }^{3}$, TAE-SUNG KIM ${ }^{3}$ and MYONG CHEOL LIM ${ }^{1,4,5}$ \\ ${ }^{1}$ Center for Gynecologic Cancer, Research Institute and Hospital, National Cancer Center; \\ Departments of ${ }^{2}$ Radiology and ${ }^{3}$ Nuclear Medicine, National Cancer Center; ${ }^{4}$ Division of Tumor Immunology, \\ Research Institute and Hospital, National Cancer Center; ${ }^{5}$ Department of Cancer Control and Policy, \\ Graduate School of Cancer Science and Policy, National Cancer Center, Goyang-si, Gyeonggi-do 10408, Republic of Korea
}

Received August 9, 2021; Accepted December 13, 2021

DOI: $10.3892 / \mathrm{mco} .2021 .2482$

\begin{abstract}
A lymphocele is a common complication that occurs following pelvic lymph node dissection. However, the complication of lymphoceles following sentinel pelvic lymph node biopsy has not been previously reported, to the best of our knowledge. A 49-year-old female patient had undergone radical hysterectomy and pelvic lymph node biopsy for stage IB1 cervical cancer 5 months previously and presented with a profuse watery vaginal discharge of $\sim 2$ liters per day. A fistula connecting the lymphocele and the vaginal stump was identified using lymphoscintigraphy and single photon emission CT/CT. Transvaginal lymphatic embolization was successfully performed through the vaginal fistulous tract, resulting in immediate reduction of the vaginal discharge. In conclusion, the case of fistula formation between pelvic lymphocele and vaginal stump was encountered at our department and was reported with a literature review. To the best of our knowledge, there are no previous reports on lymphoceles with direct communication to the vaginal mucosa, particularly following sentinel pelvic lymph node biopsy. The present study reported the case of a patient who was successfully treated for a pelvic lymphocele with direct communication to the vaginal mucosa.
\end{abstract}

\section{Introduction}

Cervical cancer is the second-most common malignant tumor in females globally and one of the leading causes of

Correspondence to: Dr Myong Cheol Lim, Center for Gynecologic Cancer, Research Institute and Hospital, National Cancer Center, 323 Ilsan-ro, Ilsandong-gu, Goyang-si, Gyeonggi-do 10408, Republic of Korea

E-mail: gynlim@gmail.com

Key words: cervical cancer, radical hysterectomy, lymphocele cancer-associated mortality (1). In 2018, with an estimated 570,000 cases and 311,000 deaths, cervical cancer ranked the fourth most frequently diagnosed cancer and the fourth leading cause of cancer-associated death in females worldwide (2). Radical hysterectomy and lymphadenectomy are standard procedures in radical surgery for cervical cancer. A lymphocele is a common complication following radical hysterectomy and pelvic lymph node dissection. The incidence ranges from $1-58 \%$. A lymphocele is a cystic mass that may occur in the retroperitoneum after pelvic lymphadenectomy. Lymphoceles may be the cause of severe morbidity, or even mortality in rare cases. In most patients affected, lymphoceles are asymptomatic, while symptomatic lymphoceles manifest with pain due to compression of adjacent structures, lymphoedema, deep vein thrombosis or inflammation (3).

At our department, a patient who underwent pelvic surgery presented with discomfort due to vaginal discharge. The patient had a rare presentation of fistula formation between a pelvic lymphocele and the vaginal stump. The present study reported on the successful treatment of this patient and provided a review of the pertinent literature. In the present article, the feasibility and result of lymphatic embolization after radical hysterectomy and sentinel pelvic lymph node biopsy were demonstrated.

\section{Case report}

The patient was a 49-year-old female who presented at the Center for Gynecologic Cancer, National Cancer Center Korea (Goyang, Republic of Korea) with a profuse vaginal discharge of $\sim 2$ 1/day at 5 months postoperatively (complication date, April 2021). The vaginal discharge was a clear, yellow-colored fluid without any admixture of blood. The patient had no symptoms other than vaginal discharge. On physical examination, the vaginal mucosa appeared normal.

The patient had undergone a robot-assisted radical hysterectomy, bilateral adnexectomy and sentinel pelvic lymph node biopsy for invasive squamous cell carcinoma of the cervix 


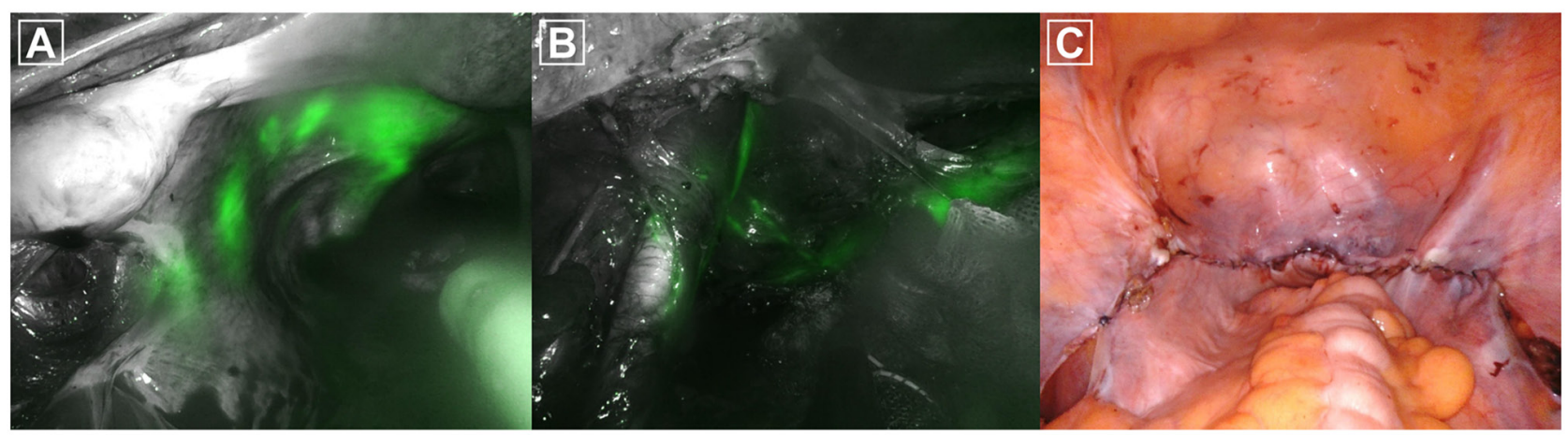

Figure 1. Intraoperative findings of radical hysterectomy and sentinel lymph node biopsy. (A) Sentinel lymph nodes in the left side of the pelvis were identified after transcervical injection of indocyanine green. (B) Sentinel lymph nodes were surgically removed for frozen section and lymphatic vessels were visualized. (C) Retroperitonealization was performed after completion of radical hysterectomy and sentinel pelvic lymph node biopsy for prevention of surgical adhesion.
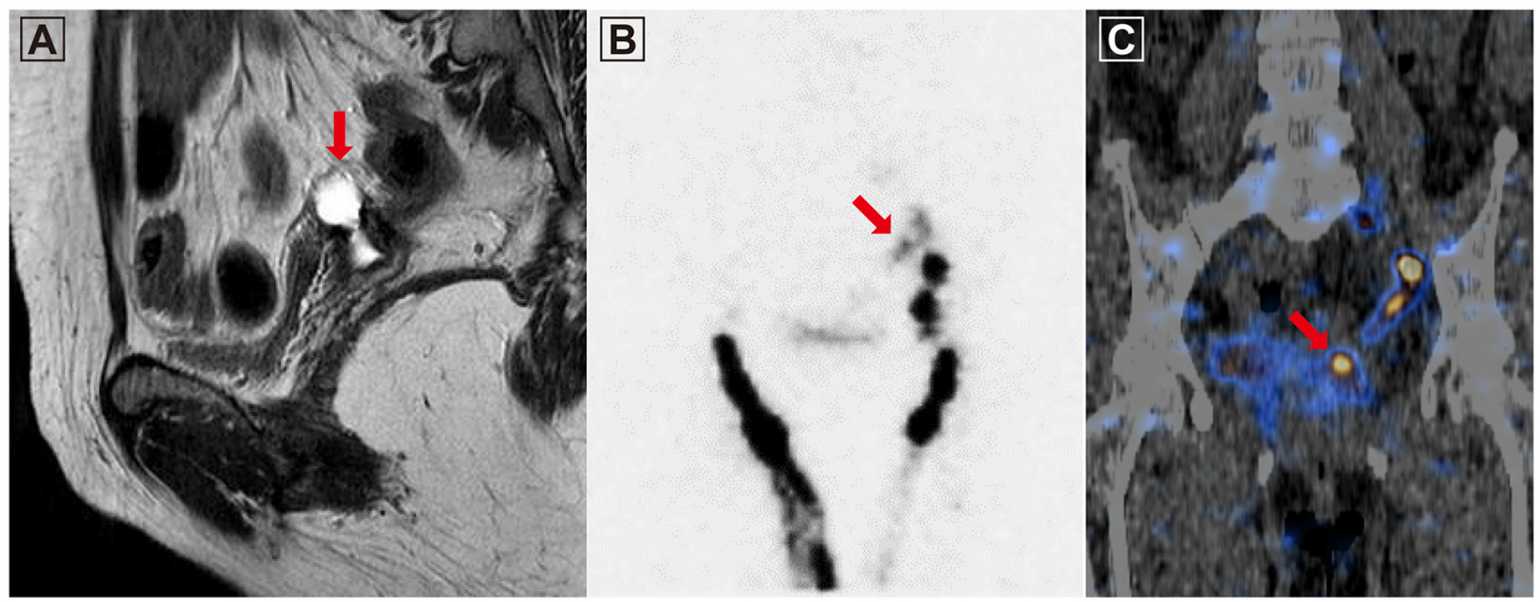

Figure 2. Images of the 49-year-old female patient with a history of radical hysterectomy and pelvic lymph node dissection. The patient presented with profuse watery vaginal discharge ( 2 1/day) at 5 months after the surgery. (A) Sagittal MRI revealed a lymphocele with communication to the vaginal stump (red arrow). (B) Lymphoscintigraphy planar image indicating lymphatic leakage in the left side of the pelvis (arrow). (C) Single-photon emission CT/CT image displaying lymphatic leakage from the lymphatic flow in the left side of the pelvis to the vaginal stump (arrow).

(International Federation of Gynecology and Obstetrics stage IB1) 5 months previously. After cervical injection of indocyanine green, sentinel lymph nodes (SLNs) were bilaterally identified among the pelvic lymph nodes. No tumor was identified on the frozen sections of the five left-sided pelvic lymph nodes and one right-sided pelvic lymph node (Fig. 1). After radical hysterectomy and sentinel pelvic lymph node biopsy, retroperitonealization (closure of the peritoneum) was performed. After hysterectomy, the vaginal stump was sutured and the peritoneum on the bladder side (anterior side) and peritoneum on the rectum (posterior side) were then sutured. Sutures were performed on both sides in the direction of the incised round ligament. The patient's postoperative course was uneventful and the patient was discharged on postoperative day 2. Histopathological examination revealed a $1.8-\mathrm{cm}$ invasive squamous cell carcinoma. There were no other risk factors, such as lymphovascular space invasion, parametrial invasion, surgical margin or lymph node complications.

As an initial investigation, the creatinine level of the vaginal discharge was evaluated and was determined to be normal $(0.51 \mathrm{mg} / \mathrm{dl})$. Pelvic MRI revealed a 2.5x1.2 cm-sized cystic lesion in the left superior region of the vaginal stump (Fig. 2A). Lymphoscintigraphy was performed using technetium-99m-labeled phytate $(4 \mathrm{mCi})$ to determine whether a fistulous connection between the pelvic lymphocele and the vaginal stump was present. A radiopharmaceutical compound was injected into the interphalangeal webs of both feet and the suspected route of lymph flow to the vaginal lumen via the lymphocele was observed in a planar image $10 \mathrm{~min}$ after the injection (Fig. 2B). Single photon emission CT/CT identified radioactivity extending from the pelvic lymphocele to the vaginal stump, followed by dynamic flow (Fig. 2C).

Lymphangiography was performed by accessing the inguinal lymph nodes on both sides. Contrast enhancement of the lymphatic ducts along the iliac chains on both sides occurred at a slow rate. The contrast medium was observed to slowly leak from the left iliac chain to the pelvic cavity after $>1 \mathrm{~h}$. The epithelial layer was not intact according to the lymphangiography/lymphoscintigraphy images (Video S1); therefore, it was not possible to perform percutaneous catheter drainage (PCD) or sclerotherapy. The leaking lymphatic vessels were embolized using coils and a glue/lipiodol mix at a ratio of 1:2 (Fig. 3). After embolization, no further discharge was observed from the vagina. The patient is currently being followed up and is doing well without any notable side effects. At three months after lymphatic embolization, a CT scan 


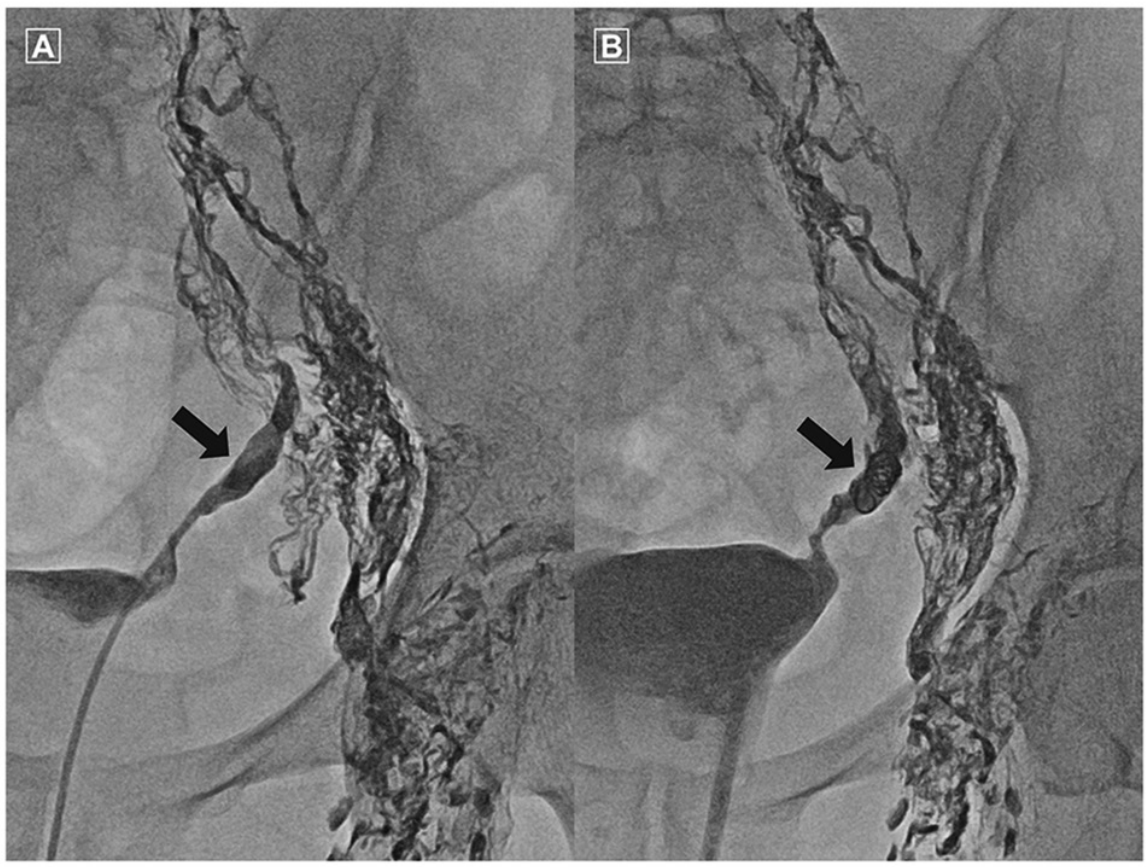

Figure 3. (A) Intranodal lymphangiography through a left inguinal lymph node indicating extravasated lipiodol (black arrow). A transvaginal approach to the left pelvic lymphocele was successfully performed. (B) N-butyl cyanoacrylate glue (1:2 mixture with lipiodol) injection (arrow) was administered to seal the lymphatic leakage. After embolization, the leakage was observed to markedly decrease without any further complications.

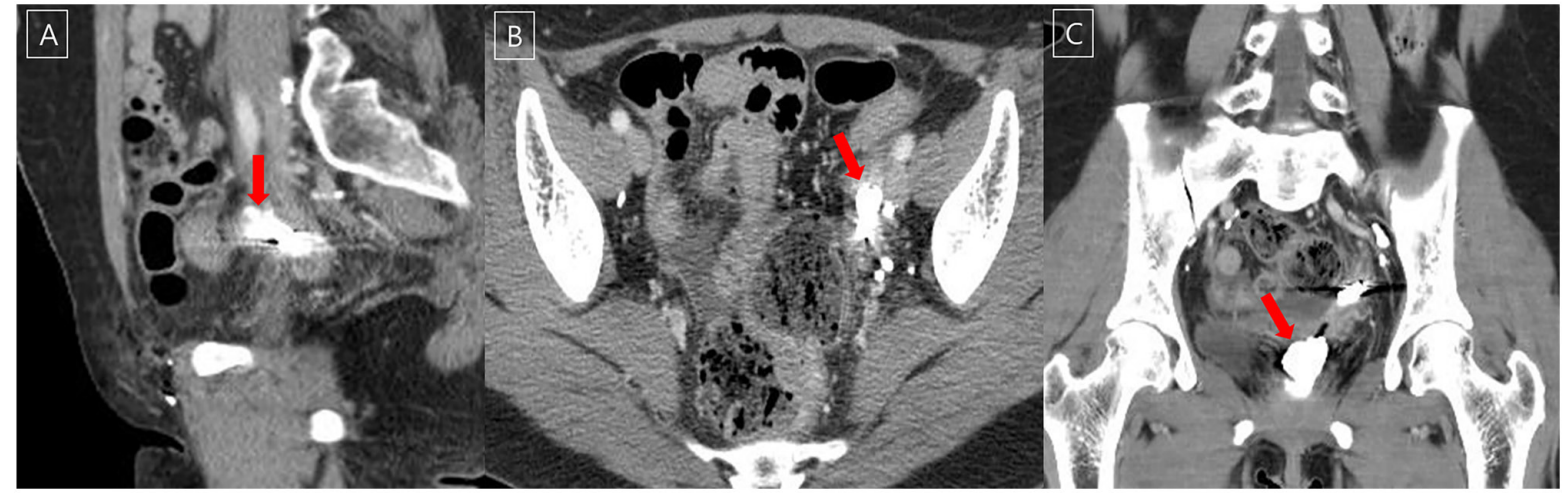

Figure 4. After lymphatic embolization, the leakage was observed to markedly decrease without any further complications. CT images indicating decreased lymphatic leakage. (A) Sagittal CT image, (B) axial CT image and (C) coronal CT image.

revealed decreased fluid collection above the vaginal stump to the left, communicating with the vagina (Fig. 4).

\section{Discussion}

A pelvic lymphocele is a collection of lymph with a thick fibrotic wall but without any epithelial lining. Lymphoceles are usually associated with extensive lymph node dissection (4). However, the patient of the present study developed a complicated pelvic lymphocele that communicated with the vaginal stump following sentinel pelvic lymph node biopsy. Even though the incidence of a lymphocele does not increase depending on the mode of peritoneal closure, in the case of the present study, retroperitonealization may have been an additional risk factor for the development of a pelvic lymphocele and a fistula to the vaginal stump due to the increased regional pressure (5). Kadanali et al (6) reported that an unreconstructed peritoneum may reduce the incidence of adhesion. Currently, it remains elusive whether retroperitonealization after pelvic lymphadenectomy is beneficial (7).

Symptomatic lymphoceles typically occur due to compression of the surrounding anatomic structures, resulting in pelvic pain, leg edema, gastrointestinal obstruction, obstructive uropathy and deep vein thrombosis. Furthermore, severe and potentially life-threatening complications, including sepsis, chylous ascites, lymphatic fistula formation and pulmonary thromboembolism, may occur (8). SLN biopsy was introduced in the field of gynecologic oncology to minimize these complications. Currently, there are no pertinent reports on complications after sentinel pelvic lymph node biopsy. To the best of our knowledge, the present study was the first to report complications following SLN biopsy. Several studies have indicated that SLN mapping in early-stage cervical cancer patients are feasible with excellent detection rates and 
sensitivity $(9,10)$. Although recent international guidelines recommend performing SLN biopsy in addition to pelvic lymph node dissection, SLN biopsy alone is not the gold standard yet due to a lack of prospective evidence, particularly in terms of long-term oncological safety. For the diagnosis of cervical cancer stage I, a pelvic lymph node dissection other than SLN biopsy is available. In the case of the present study, only SLN biopsy was performed. There was no lesion suspicious of lymph node metastasis in the preoperative MRI and there was no uptake on positron emission tomography/CT. All SLN biopsy specimens, even those from intraoperative frozen biopsy, were reported as negative. SLN was performed according to the cervical cancer treatment guidelines from the National Comprehensive Cancer Network (11). No other diagnostic method was available.

The diagnosis of a lymphocele is based on imaging findings. On MRI, lymphoceles appear as lobulated, hyperintense structures on T2-weighted images with imperceptible walls and negligible wall enhancement on postcontrast T1-weighted images. There is no definitive consensus on the treatment of pelvic lymphoceles. Conservative treatment is attempted first and follow-up is performed using CT or ultrasonography to identify lesion regression. Surgical marsupialization by open or laparoscopic surgery or percutaneous catheter drainage are options to manage pelvic lymphoceles (4). In the last two decades, interventional radiology has become an important strategy in the treatment of pelvic lymphoceles. PCD may be combined with transcatheter ethanol sclerotherapy to augment the therapeutic efficiency. PCD is an easy, safe and successful treatment method in $80-90 \%$ of cases. However, it takes longer for results to be achieved (10-20 days, as reported in the literature). In addition, percutaneous techniques carry a risk for potential infection (4,12-14). Recently, lymphatic embolization using N-butyl cyanoacrylate glue was performed to safely and effectively treat postoperative pelvic lymphoceles, for which PCD is insufficient (15).

Among the aforementioned treatments, PCD and sclerotherapy are relatively easy to perform. Lymphangiography is able to determine how much radioactive material has been removed from the injection site, how much has been absorbed into the surrounding lymph nodes, whether there is a delay or blockage of lymph drainage or whether dermal reflux occurs. Lymphangiography and lymphatic embolization are invasive procedures including incision or puncture and there are inherent risks such as infection, pain and lipiodol extravasation during injection (16). Even in the presence of multiple leakage points, treatment is possible if the epithelial layer of the cavity is adherent. However, patients with high-flow leakages have a higher chance of recurrence, lowering the chance of a cure as cavity adhesion slowly occurs. Since pedal lymphoscintigraphy indicated that the epithelial layer was not intact and that a lymphocele had not completely formed in the present case, it would have been difficult to insert a percutaneous catheter for drainage and/or attempt sclerotherapy. For successful lymphatic embolization, leaking lymphatic vessels must be obliterated. However, it was not possible to clearly localize the point of leakage in the present case and a slow leak from the external iliac chain was identified on lymphoscintigraphy and lymphangiography. The larger lymph nodes around the leak may be used for glue injection to achieve lymphatic obstruction. However, no large lymph nodes were observed in the present case. Alternatively, transvaginal lymphatic embolization was attempted and performed successfully once.

In conclusion, transvaginal lymphatic embolization is a rapid and reliable method that may be used to directly visualize the tract (from the vaginal stump to the lesion in the present case) prior to lymphatic blockage. The effect of treatment may also be immediately confirmed.

\section{Acknowledgements}

Not applicable.

\section{Funding}

No funding was received.

\section{Availability of data and materials}

The datasets used and/or analyzed during the current study are available from the corresponding author on reasonable requests.

\section{Authors' contributions}

YJL, IJL, SP, TSK and MCL were involved in the conception and design of the current study. YJL wrote the manuscript. IJL, SP, TSK and MCL supervised the study. YJL and MCL confirm the authenticity of all the raw data. All authors have read and approved the final version of the manuscript.

\section{Ethics approval and consent to participate}

The Ethics Committee of National Cancer Center Korea (Goyang, Korea) approved this case report. The patient provided written informed consent.

\section{Patient consent for publication}

The patient provided informed consent for the publication of her case including data and images.

\section{Competing interests}

The authors declare that they have no competing interests.

\section{References}

1. Mattiuzzi C and Lippi G: Cancer statistics: A comparison between World health organization (WHO) and global burden of disease (GBD). Eur J Public Health 30: 1026-1027, 2020.

2. Bray F, Ferlay J, Soerjomataram I, Siegel RL, Torre LA and Jemal A: Global cancer statistics 2018: GLOBOCAN estimates of incidence and mortality worldwide for 36 cancers in 185 countries. CA Cancer J Clin 68: 394-424, 2018.

3. Weinberger V, Cibula D and Zikan M: Lymphocele: Prevalence and management in gynecological malignancies. Expert Rev Anticancer Ther 14: 307-317, 2014.

4. Karcaaltincaba $M$ and Akhan O: Radiologic imaging and percutaneous treatment of pelvic lymphocele. Eur J Radiol 55: 340-354, 2005.

5. Cheong YC, Bajekal N and Li TC: Peritoneal closure-to close or not to close. Hum Reprod 16: 1548-1552, 2001. 
6. Kadanali S, Erten O and Kucukozkan T: Pelvic and periaortic pertioneal closure or non-closure at lymphadenectomy in ovarian cancer: Effects on morbidity and adhesion formation. Eur J Surg Oncol 22: 282-285, 1996.

7. Ai W, Liang Z, Li F and $\mathrm{Yu} \mathrm{H}$ : Internal hernia beneath superior vesical artery after pelvic lymphadenectomy for cervical cancer: A case report and literature review. BMC Surg 20: 312, 2020.

8. Clarke-Pearson DL, Synan IS and Creasman WT: Significant venous thromboembolism caused by pelvic lymphocysts: Diagnosis and management. Gynecol Oncol 13: 136-143, 1982.

9. Cibula D and McCluggage WG: Sentinel lymph node (SLN) concept in cervical cancer: Current limitations and unanswered questions. Gynecol Oncol 152: 202-207, 2019.

10. Kim JH, Kim DY, Suh DS, Kim JH, Kim YM, Kim YT and Nam JH: The efficacy of sentinel lymph node mapping with indocyanine green in cervical cancer. World J Surg Oncol 16: 52, 2018.

11. Abu-Rustum NR, Yashar CM, Bean S, Bradley K, Campos SM, Chon HS, Chu C, Cohn D, Crispens MA, Damast S, et al: NCCN guidelines insights: Cervical cancer, version 1.2020. J Natl Compr Canc Netw 18: 660-666, 2020.
12. vanSonnenberg E, Wittich GR, Casola G, Wing VW, Halasz NA, Lee AS and Withers C: Lymphoceles: Imaging characteristics and percutaneous management. Radiology 161: 593-596, 1986.

13. Aronowitz J and Kaplan AL: The management of a pelvic lymphocele by the use of a percutaneous indwelling catheter inserted with ultrasound guidance. Gynecol Oncol 16: 292-295, 1993.

14. Kim JK, Jeong YY, Kim YH, Kim YC, Kang HK and Choi HS Postoperative pelvic lymphocele: Treatment with simple percutaneous catheter drainage. Radiology 212: 390-394, 1999.

15. Chu HH, Shin JH, Kim JW, Noh SY, Yang WJ and Park S: Lymphangiography and lymphatic embolization for the management of pelvic lymphocele after radical prostatectomy in prostatic cancer. Cardiovasc Intervent Radiol 42: 873-879, 2019.

16. Lee EW, Shin JH, Ko HK, Park J, Kim SH and Sung KB Lymphangiography to treat postoperative lymphatic leakage: A technical review. Korean J Radiol 15: 724-732, 2014. 